\title{
Perinatal Tobacco Smoke Exposure Increases Vascular Oxidative Stress and Mitochondrial Damage in Non-Human Primates
}

\author{
David G. Westbrook • Peter G. Anderson • \\ Kent E. Pinkerton $\cdot$ Scott W. Ballinger
}

Published online: 29 July 2010

(C) The Author(s) 2010. This article is published with open access at Springerlink.com

\begin{abstract}
Epidemiological studies suggest that events occurring during fetal and early childhood development influence disease susceptibility. Similarly, molecular studies in mice have shown that in utero exposure to cardiovascular disease (CVD) risk factors such as environmental tobacco smoke (ETS) increased adult atherogenic susceptibility and mitochondrial damage; however, the molecular effects of similar exposures in primates are not yet known. To determine whether perinatal ETS exposure increased mitochondrial damage, dysfunction and oxidant stress in primates, archived tissues from the non-human primate model Macaca mulatta ( $M$. mulatta) were utilized. M. mulatta were exposed to low levels of ETS $\left(1 \mathrm{mg} / \mathrm{m}^{3}\right.$ total suspended particulates) from gestation (day 40) to early childhood (1 year), and aortic tissues were assessed for oxidized proteins (protein carbonyls), antioxidant activity (SOD), mitochondrial function (cytochrome oxidase), and mitochondrial damage (mitochondrial DNA damage). Results revealed that perinatal ETS exposure resulted in
\end{abstract}

D. G. Westbrook · P. G. Anderson · S. W. Ballinger ( $₫)$ Department of Pathology, Division of Molecular and Cellular Pathology, University of Alabama at Birmingham, VH G019F, 1530 3rd Avenue S., Birmingham, AL 35294-0019, USA e-mail: sballing@uab.edu

D. G. Westbrook · S. W. Ballinger

Center for Free Radical Biology, University of Alabama at

Birmingham, Birmingham, AL, USA

K. E. Pinkerton

Center for Health and the Environment, University of California,

Davis, CA, USA

S. W. Ballinger

Department of Environmental Health Sciences, University

of Alabama at Birmingham, Birmingham, AL, USA significantly increased oxidative stress, mitochondrial dysfunction and damage which were accompanied by significantly decreased mitochondrial antioxidant capacity and mitochondrial copy number in vascular tissue. Increased mitochondrial damage was also detected in buffy coat tissues in exposed M. mulatta. These studies suggest that perinatal tobacco smoke exposure increases vascular oxidative stress and mitochondrial damage in primates, potentially increasing adult disease susceptibility.

Keywords Tobacco $\cdot$ Cigarette smoke $\cdot$ Mitochondria . Macaque $\cdot$ mtDNA $\cdot$ Oxidative stress $\cdot$ Vascular

\section{Introduction}

Cardiovascular disease (CVD) development generally begins decades before its clinical manifestations are apparent. Epidemiological studies of CVD risk factors suggest that events occurring during fetal and early childhood development (e.g. exposure to a risk factor) may influence disease susceptibility, and in this regard, studies have shown that in utero exposure to CVD risk factors such as environmental tobacco smoke (ETS) and hypercholesterolemia convey increased adult atherogenic susceptibility in exposed animals $[1,2]$. In humans, it has been estimated that over half the children aged 4-11 have cotinine in their blood, suggesting that exposure to nicotine or cigarette smoke is a regular occurrence [3]. Similarly, it has also been estimated that $22 \%$ of children under the age of 18 are regularly exposed to cigarette smoke in the home [3]. Studies have also revealed that while many female smokers will abstain from smoking during pregnancy, $13.2 \%$ of U.S. women continue to smoke during pregnancy [4]. Consequently, a significant number of children are exposed 
to cigarette smoke during gestation and early childhood, and while there have been numerous reports that associated low birth weights and delayed developmental effects in children associated with tobacco smoke exposure, the molecular effects of these exposures are not yet clearly known [5, 6].

It has been previously shown that a relationship exists between fetal exposure to ETS, mitochondrial damage and adult atherosclerotic lesion development in mice [1, 2]. These effects were not associated with significant changes in LDL or HDL cholesterol levels in the mother or offspring, but instead, were associated with increased mitochondrial damage and oxidative stress in exposed offspring [1]. While these findings suggest that both increased atherosclerotic lesion formation and mitochondrial damage are associated with in utero exposure to ETS, molecular studies demonstrating similar effects in humans or nonhuman primates are currently lacking. Herein, molecular data are presented from archived tissues from the nonhuman primate model Macaca mulatta ( $M$. mulatta). For these studies, archived aortic and blood tissues were utilized from $M$. mulatta that were exposed to low levels of ETS during gestation and early childhood were assessed for mitochondrial function, antioxidant activity, oxidative stress and mtDNA damage. Results reveal that exposure to even relatively low ETS dosages during gestation and early childhood significantly impacted vascular morphology, oxidative stress, antioxidant activity, mitochondrial function and damage. In addition, differences in mitochondrial damage could also be detected in blood, suggesting these effects were not isolated to the vascular tissues. Consequently, these studies provide molecular evidence for increased oxidative stress and mitochondrial damage in primate aortic tissues associated with developmental ETS exposure.

\section{Methods}

\section{Monkeys}

Archived aortic and blood (buffy coats) tissues from three ETS-exposed (gestation day 40 until 1 year of age) and three age-matched control (unexposed) male monkeys were utilized. Briefly, normally cycling, adult female rhesus monkeys were bred and identified as pregnant via ultrasound (gestation day 40), using established methods [7]. Pregnancy in the rhesus monkey is divided into trimesters by 55 day increments with 0-55 days gestation representing the first trimester, 56-110 days gestation representing the second trimester, and 111-165 days gestation the third trimester (term $165 \pm 10$ days). All fetuses were sonographically assessed to confirm normal growth and development prior to assignment to the study. All measures were compared to normative growth curves and developmental parameters for rhesus fetuses [8]. Animals were handled in accordance with standards established by the US Animal Welfare Acts as set forth in the National Institutes of Health Guidelines and by the University of California, Davis, Animal Care and Use Committee.

\section{Exposure Regimen and Generation of ETS}

Commencing at gestation day 40, pregnant dams were exposed to ETS or FA for $6 \mathrm{~h} /$ day, 5 days/week. All animals were closely monitored during exposure for health and pregnancy status. Upon birth, both dam and infant were housed in the same exposure chamber. The exposure system and monitoring methods have been previously described in detail [9]. Briefly, ETS was generated by a smoke exposure system (Teague Enterprises, Davis, CA) using IR4F conditioned cigarettes from the Tobacco and Health Research Institute of the University of Kentucky. Sidestream smoke was drawn into a conditioning chamber where it was aged, diluted, and then further diluted as it passed into the exposure chambers to produce total suspended particulate (TSP) concentrations of $1.0 \mathrm{mg} / \mathrm{m}^{3}$, 4-5 ppm carbon monoxide, and 200-300 $\mu \mathrm{g} / \mathrm{m}^{3}$ nicotine. This level of exposure is highly reminiscent of ETS concentrations found in homes or the workplace where smoking is permitted. The exposure chambers were stainless steel and glass Hinners-type and $4.2 \mathrm{~m}^{3}$ in size. Each chamber has an air capacity of $3.5 \mathrm{~m}^{3}$.

\section{Tissue Collection}

Necropsies were performed under sterile conditions with full protective clothing at the California Regional Primate Research Center. Under the direction of veterinarians and pathology staff, each animal was euthanized under humane conditions. The aorta was removed and perfused with cold PBS buffer, and snap frozen in liquid nitrogen, and stored at $-80^{\circ} \mathrm{C}$.

\section{Histology}

Frozen archived tissues were thawed and fixed in $10 \%$ neutral-buffered formalin, embedded in paraffin, sectioned, and stained with hematoxylin-eosin. Slides were provided (blinded) to an experienced cardiovascular pathologist for scoring. Coded slides were examined and given a grade using a scale with 0 being morphologically normal endothelium with no subintimal cells and a score of 3 representing endothelial disruption and significant accumulation 
of cells within the subintimal region. Grades 1 or 2 were assigned to sections that were intermediate.

\section{SOD Activity}

SOD activity was quantified using the cytochrome $\mathrm{C}$ reduction assay. This assay is based upon the ability of SOD to inhibit the reduction of cytochrome $\mathrm{C}$ by $\mathrm{O}_{2}{ }^{-}$ generated by xanthine/xanthine oxidase [10]. Increased SOD activity results in inhibition of cytochrome $\mathrm{C}$ reduction, reflected by decreased absorbance at $550 \mathrm{~nm}$. Cyanide and azide ( $3 \mathrm{mM} \mathrm{KCN}, 3 \mathrm{mM} \mathrm{NaN}_{3}$ ) were used to inhibit cytoplasmic and extracellular SOD, allowing for direct measurement of mitochondrial SOD (SOD2) activity. Activity was determined from a standard curve generated using known amounts of SOD2 (Sigma S-5639).

\section{Quantitative PCR (QPCR) for Evaluating DNA Damage}

MtDNA damage was quantified by QPCR, which is based upon the principle that DNA lesions will block the polymerase and therefore will lead to a decrease in target amplification. This assay has been used to quantify DNA damage both in vitro [11] and in vivo [12]. For these studies, genomic DNA was extracted (Qiagen) from archived tissues (aorta, buffy coat) harvested from male $M$. mulatta exposed to cigarette smoke $(N=3)$ or filtered air $(N=3)$, or human buffy coats collected from male, age-matched smokers $(N=8$; mean age $40.25 \pm 2.27$ years $)$ and non-smokers $(N=5$; mean age $41.6 \pm 1.47$ years). Human buffy coat were isolated from whole blood as previously described [13]. Extracted DNA was quantified fluorescently (Picogreen, Invitrogen), and $15 \mathrm{ng}$ was used to amplify a $16 \mathrm{~kb}$ mtDNA QPCR product using primer set MQND5For/ MQND4Rev: $5^{\prime}$-caaccctcatctccetgacccttc/cgtgtgaaggggggtt ttatattga- $3^{\prime}$ in $M$. mulatta and previously reported primers in humans [11]. Each QPCR reaction experiment included a standard QPCR using a serial dilution of 30, 15, 7.5, 3.75, $1.875,0.937$ and $0 \mu \mathrm{g}$ of control genomic DNA (M. mulatta or human) as template to verify quantitative amplification. Potential differences in mtDNA copy numbers were normalized by using a short $(0.2 \mathrm{~kb})$ QPCR reaction product using primers MQmtstFor/MQmtstRev: 5'-caactatagcaacagccttcata/aa ctaggtctgttccaatgtatg- $3^{\prime}$. DNA damage was quantified by dividing the $16 \mathrm{~kb}$ product by the corresponding $0.2 \mathrm{~kb}$ product, and comparing the normalized ETS-exposed values to normalized unexposed control values, resulting in a relative amplification ratio ( $A_{\mathrm{ETS}}$ ) $A_{\text {unexposed }}$ ). Assuming a random distribution of lesions and using the Poisson equation $\left[f(x)=e^{\lambda} \lambda^{x} / x\right.$ !, where $\lambda=$ the average lesion frequency] for the non-damaged templates (zero class; $x=0$ ), the average lesion frequency per DNA strand is determined; $\lambda=-\ln A_{\mathrm{ETS}} / A_{\text {unexposed }}[11,12,14]$.

To assess the levels of specific DNA lesions recognized by formamidopyrimidine (Fapy) DNA glycosylase (Fpg, New England BioLabs), which cleaves DNA at specific lesions such as 7, 8-dihydro-8-oxoguanonine, 8-oxoadenine, fapy-guanine, methy-fapy-guanine, fapy-adenine, and 5-hydroxy-cytosine, $1 \mu \mathrm{g}$ of genomic aortic DNA was digested with Fpg (12 units) or sham treated (to confirm digestion) overnight at $37^{\circ} \mathrm{C}$. Next, DNA was re-extracted (Qiagen), and QPCR performed on $15 \mathrm{ng}$ genomic DNA. Levels of Fpg-recognized DNA adducts were calculated to by comparing the DNA damage results from the ETSexposed animals to unexposed controls, as described above. Overall levels of Fpg detectable lesions were then expressed in ETS-exposed animals as increase in Fpg detectable lesions/16 kb relative to Fpg-treated, unexposed controls.

\section{Mitochondrial Number Determination}

Mitochondrial numbers were estimated based upon the amount of mtDNA present in $15 \mathrm{ng}$ of genomic DNA. Because the number of mtDNAs present within a mitochondrion remains stable (5-10 copies per mitochondrion), total mitochondrial numbers were estimated via amplification of a short region of mtDNA using primers MQmtstFor/ MQmtstRev: $5^{\prime}$-caactatagcaacagccttcata/aa ctaggtctgttcc aatgtatg- $3^{\prime}$ from equal amounts of genomic DNA between samples. Amplification products were then quantified and expressed relative to control tissue.

\section{SOD2 and Anti-Nitrotyrosine Immunoblotting}

Thirty micrograms of homogenized primate aorta was denatured in SDS-PAGE sample buffer by heating at $95^{\circ} \mathrm{C}$ for $10 \mathrm{~min}$. Samples were separated on an 18-well 4-12\% bis-tris gel (BioRad 03450124) using MES as running buffer (BioRad 161-0789). Electrophoretic separation of the proteins was conducted for an hour at $100 \mathrm{~V}$ or until leader dye reached bottom of the gel. Separated proteins were transferred to nitrocellulose membrane ( 0.45 micron) for $12 \mathrm{~h}$ (overnight), $150 \mathrm{~mA}, 4^{\circ} \mathrm{C}$. The blots were ponceau stained, imaged, and then blocked in 3\% NFDM (BioRad) in TBS for $3 \mathrm{~h}$ at room temp. Blots were rinsed once with tris-buffered saline (TBS) and probed with either primary rabbit anti-MnSOD (Fitzgerald \#RDI-RTSODMAbr) 1:5,000, or primary goat anti-nitrotyrosine (Biodesign K97520G) $1: 1,000$, overnight at $4^{\circ} \mathrm{C}$. Blots were washed four times 10 min each with TBS-Tween (TBST), and then probed with HRP-conjugated goat anti-rabbit (Sigma) 1:10,000, or rabbit anti-goat (Sigma) 1:20,000, for $2 \mathrm{~h}$. 
Blots were developed using ECL-plus (GE RPN2132) and imaged using a Storm 840. Lanes were quantitated using ImageQuant software. A control sample was run on multiple blots to enable comparisons and data normalization between blots.

\section{DNP Treatment and Immunoblotting}

Homogenized primate aorta samples were derived according to protocol [15]. Briefly, to 100 micrograms protein, one volume $12 \%$ SDS was added. To half protein sample (50 micrograms), two volumes of 2,4-Dinitrophenylhydrazine (D199303) solution was then added and allowed to incubate for ten minutes at room temperature. Solution was brought to neutrality using $2 \mathrm{M}$ tris with $3 \%$ glycerol. Volume reduced as necessary by speed vacuum to load onto 18-well 4-12\% bis-tris gel (BioRad 0345-0124) using MES as running buffer (BioRad 161-0789). Electrophoretic separation of the proteins was conducted for an hour at $100 \mathrm{~V}$ or until leader dye reached bottom of the gel. Separated proteins were transferred to nitrocellulose membrane $(0.45$ micron) for $12 \mathrm{~h}$ (overnight), $150 \mathrm{~mA}, 4^{\circ} \mathrm{C}$. The blots were blocked in $3 \%$ NFDM (BioRad) in TBS for $3 \mathrm{~h}$ at room temp. Blots were rinsed once with tris-buffered saline (TBS) and probed with primary rabbit anti-DNP (Sigma D9656) 1:1,000 for $2 \mathrm{~h}$ at room temp. Blots were washed four times $10 \mathrm{~min}$ each with TBS-Tween (TBST), and then probed with HRP-conjugated goat anti-rabbit (Sigma) 1:20,000 for $2 \mathrm{~h}$. Blots were developed using ECL-plus (GE RPN2132) and imaged using a Storm 840. Lanes were quantitated using ImageQuant software.

Isolation of Mitochondria and Cytochrome c Oxidase Activity

Complex IV activity was determined from previously prepared, frozen mitochondrial isolates. Briefly, mitochondria were isolated from aortic tissue homogenized in isolation buffer on ice as previously described [16], centrifuged at $1,200 \mathrm{~g}\left(4^{\circ} \mathrm{C}, 20 \mathrm{~min}\right)$, and supernatant centrifuged at $13,000 \mathrm{~g}\left(4^{\circ} \mathrm{C}, 20 \mathrm{~min}\right)$ to pellet mitochondria which were resuspended in $0.2-\mathrm{ml}$ isolation buffer and stored at $-80^{\circ} \mathrm{C}$ until further use. Cytochrome c oxidase activity was determined by following the oxidation of reduced cytochrome $\mathrm{c}$ at $550 \mathrm{~nm}$ [16]. Briefly, reduced cytochrome c was prepared using ascorbate and its concentration determined. The oxidation of reduced cytochrome $\mathrm{c}$ was followed in the presence of the sample and potassium phosphate buffer for $3-4 \mathrm{~min}$ at $37^{\circ} \mathrm{C}$. One unit of enzyme activity is defined as by the enzymatic conversion of 1 micromole of substrate per minute. Unit enzyme activity is calculated using the first derivative of the Beers Lambert law, which is $(1 \mathrm{unit} / \mathrm{mL}$ or umol reduced cyt $\mathrm{C}$ conversion $/ \mathrm{min} / \mathrm{mL})=($ change in
Absorbance at $550 \mathrm{~nm} / \mathrm{min}) /($ millimolar extinction coefficient, with the unit of $\left.\mathrm{mmol} / \mathrm{l}^{-1} \mathrm{~cm}^{-1}\right)$. This $\mathrm{U} / \mathrm{ml}$ enzyme activity is then normalized by $\mathrm{mg} / \mathrm{ml}$ protein concentration to yield $\mathrm{U} / \mathrm{mg}(\mathrm{mU} / \mathrm{mg})$ protein.

\section{Protein Quantification}

Protein quantification from tissue homogenates and mitochondrial preparations were done by Lowry assay [17].

\section{Statistical Analyses}

Results from the quantitative studies are expressed in terms of mean and standard error (mean \pm SE). Statistical ANOVA and Student-Newman-Keuls Test (when appropriate) with Bonferroni adjustment for number of comparisons were performed using SAS software to assess the effects of exposure regimen on each parameter.

\section{Results}

To determine the effects of perinatal exposure to ETS on vascular oxidative and nitrative stress, oxidized and nitrated protein levels were determined via quantification of protein carbonylation and 3-nitrotyrosine levels in aortic homogenates, respectively. Protein carbonylation is determined via derivatization of the carbonyl group with 2,4-dinitrophenylhydrazine (DNPH), leading to the formation of stable dinitrophyenyl (DNP) hydrazone products which can be immunodetected and quantified. Figure 1a shows that the levels of DNP-labeled proteins were significantly increased in the ETS-exposed monkeys compared to the unexposed controls, suggesting that oxidized proteins were significantly higher in aortas from monkeys exposed to ETS perinatally compared to unexposed controls. Similarly, Fig. $1 \mathrm{~b}$ reveals that 3-NT levels were significantly increased with perinatal ETS exposure as well in M. mulatta compared to controls. To determine whether ETS exposure specifically impacted the mitochondrion in vascular tissue, as reported in mouse models [1, 12], mitochondrial SOD (MnSOD, SOD2) activities were determined. Figure 2 a shows that $M$. mulatta sustaining perinatal ETS exposure had significantly lower SOD2 activity compared to unexposed controls. These decreases in SOD2 activity were accompanied with significantly decreased SOD2 protein levels in the aortic tissues from M. mulatta exposed to perinatal ETS compared to controls (Fig. 2b).

To determine whether perinatal ETS exposure impacted mitochondrial integrity, quantitative PCR was performed on aortic tissue collected from $M$. mulatta. Figure 3 illustrates that perinatal ETS exposure resulted in significantly increased levels of mtDNA damage in aortic tissues 

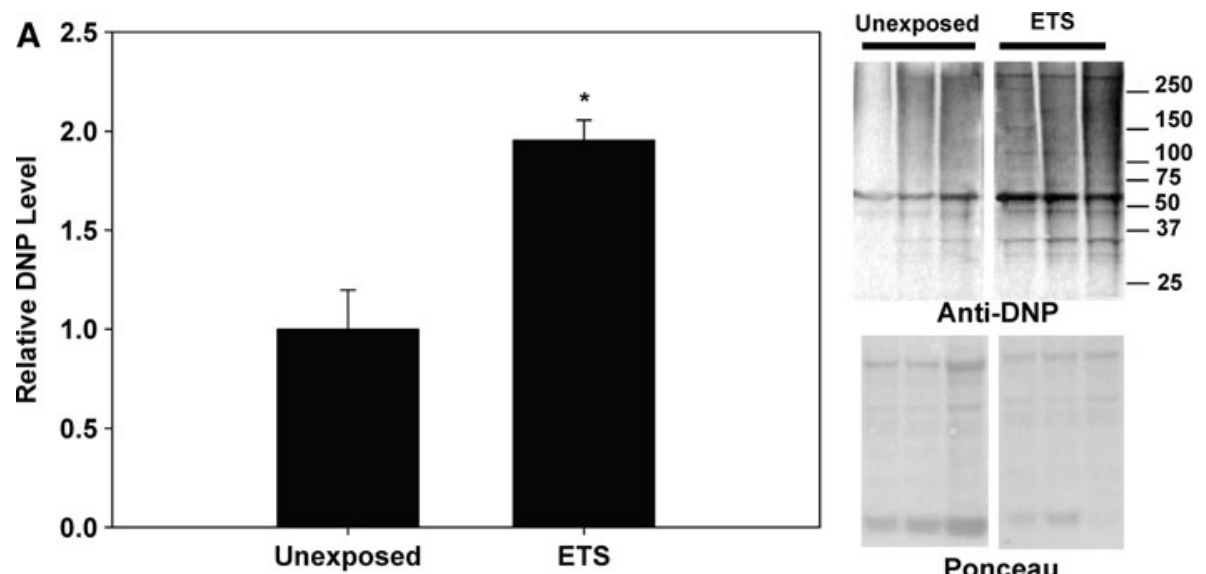

\section{Anti-DNP}

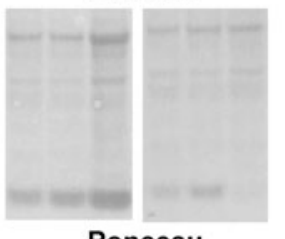

Ponceau
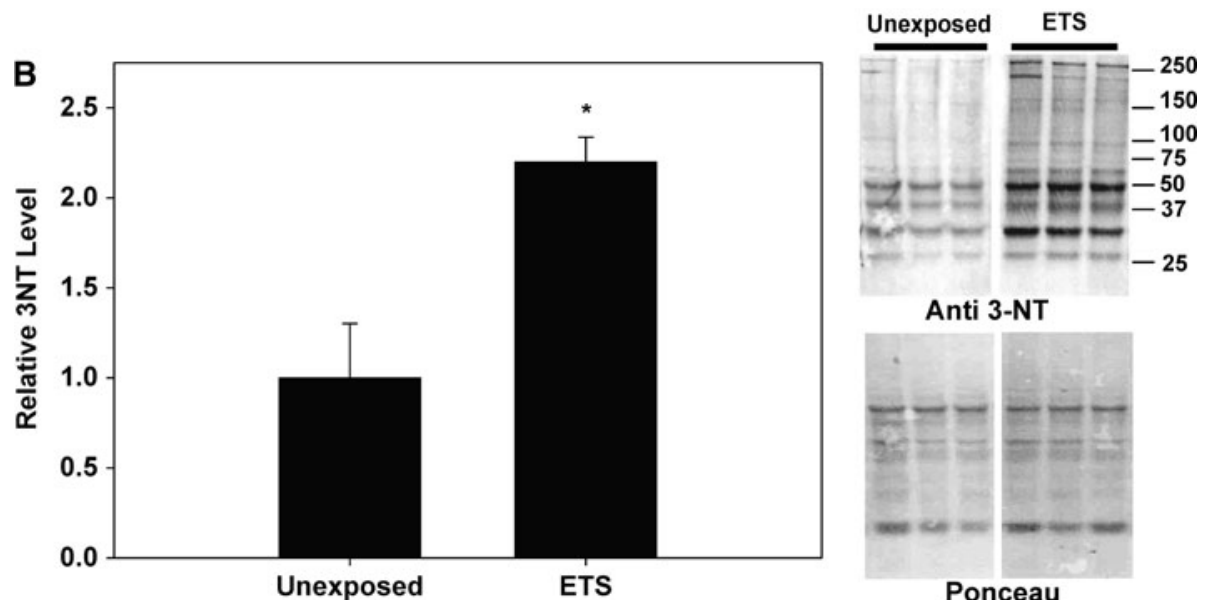

Anti 3-NT

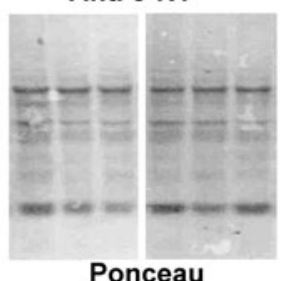

Fig. 1 Relative levels of 2,4 dinitrophenylhydrazine (DNP) and 3-nitrotyrosine (3-NT) in aortic homogenates isolated from $M$. mulatta. a Aortic homogenates were derivatized based on the reaction of carbonyl groups with 2,4-dinitrophenylhydrazine ( $D N P H)$ to form 2,4-dinitrophenylhydrazone derivatives, subjected to electrophoresis, and immunoblotted with anti-2,4 DNP antibody ( $n=3 /$ group). The panels to the right of the graph provide a typical anti-DNP immunoblot (upper panel) and ponceau-stained gel (lower panel, total protein stain) for unexposed and perinatal ETS-exposed animals (lanes 1-3 and 4-6, respectively). b Aortic protein homogenates were subjected to electrophoresis and immunoblotted with anti-3NT antibody ( $n=3$ /group). The panels to the right of the graph provide a typical anti-3NT (upper panel) immunoblot and ponceau-stained gel (lower panel, total protein stain) for unexposed and perinatal ETSexposed animals (lanes 1-3 and 4-6, respectively). Unexposed, M. mulatta age-matched control; ETS, M. mulatta exposed to $1 \mathrm{mg} / \mathrm{m}^{3}$ ETS during gestation to 1 year of age. Asterisks indicate significant difference $(P<0.05)$ from unexposed $M$. mulatta

perinatally to ETS, and also from age-matched human smokers and non-smokers. Figure 4a shows that perinatal cigarette smoke exposure increased mtDNA damage in M mulatta buffy coat DNA (less product in the "long" row on inset reflects more mtDNA damage). Figure $4 \mathrm{~b}$ reveals that human smokers also had significantly increased mtDNA damage compared to non-smokers, consistent with the notion that tobacco smoke exposure induces mitochondrial damage in humans. Interestingly, perinatal tobacco smoke exposure was also accompanied by a significant decrease in total mtDNA copy number in aorta (Fig. 5), but not blood, in M. mulatta.

To determine whether these changes in mtDNA integrity were associated with altered mitochondrial function in M. mulatta exposed to perinatal ETS, cytochrome oxidase 

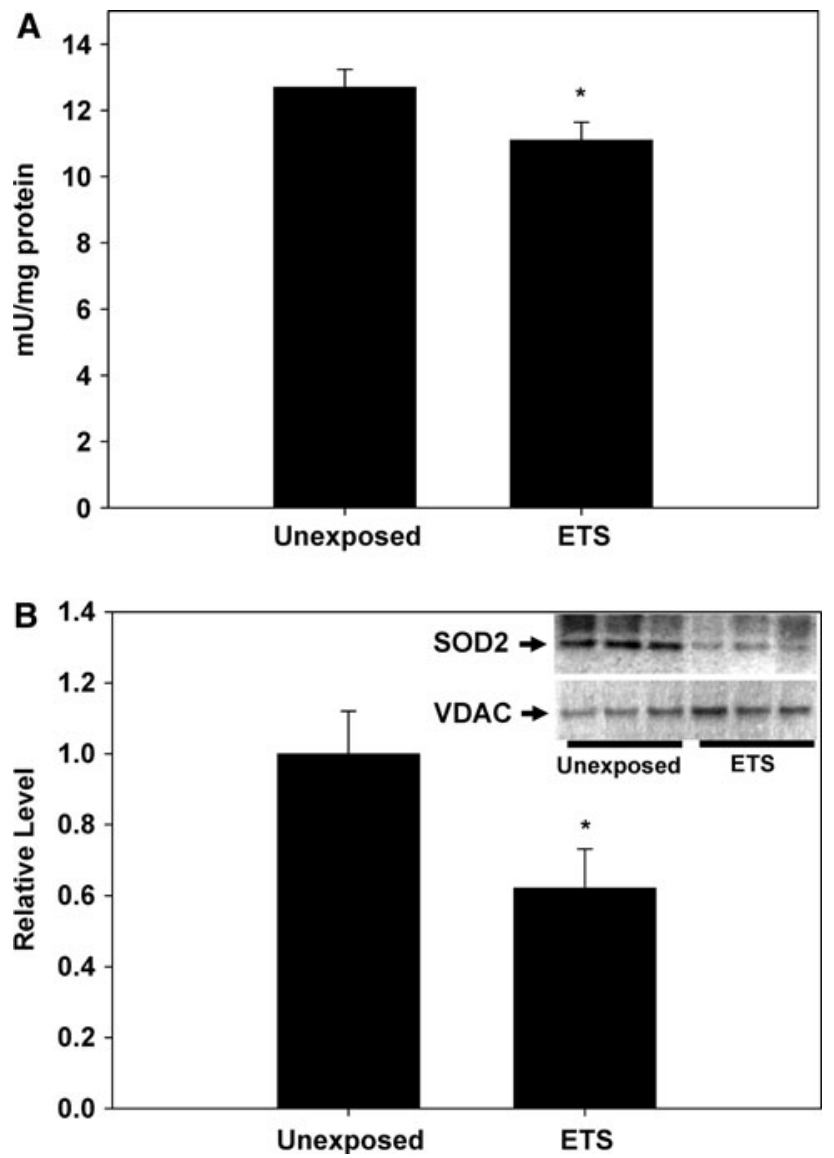

Fig. 2 Mitochondrial SOD (SOD2) activity and protein levels in M. mulatta aorta. a SOD2 activity in exposed (ETS) and unexposed control M. mulatta. b Thirty micrograms of protein from aortic homogenates were subjected to electrophoresis and immunoblotted with anti-SOD2 or VDAC (for normalization) antibodies $(n=3$ / group) and quantified densitometrically. Inset SOD2 and VDAC immunoblots from unexposed and ETS-exposed animals (lanes $1-3$ and 4-6, respectively). Unexposed, $M$. mulatta agematched control; ETS, $M$. mulatta exposed to $1 \mathrm{mg} / \mathrm{m}^{3}$ ETS during gestation to 1 year of age. Asterisks indicate significant difference $(P<0.05)$ from unexposed $M$. mulatta

(complex IV) activity was determined from aortic samples. Figure 6 reveals that mitochondrial function was significantly declined in M. mulatta sustaining perinatal exposure to ETS compared to unexposed controls.

Finally, to determine whether these observed changes in molecular biology were also accompanied by differences in histology, archived tissues were fixed, sectioned and stained with hematoxylin-eosin, and provided to a trained pathologist in a blinded fashion for scoring. Results showed that perinatal exposure to ETS was associated with significantly increased numbers of cells within the subintima, indicative of early atherogenic changes in the abdominal aorta compared to age-matched controls (Fig. 7). Consequently, both changes at the molecular and cellular levels appear to be associated with perinatal ETS exposure in $M$. mulatta.
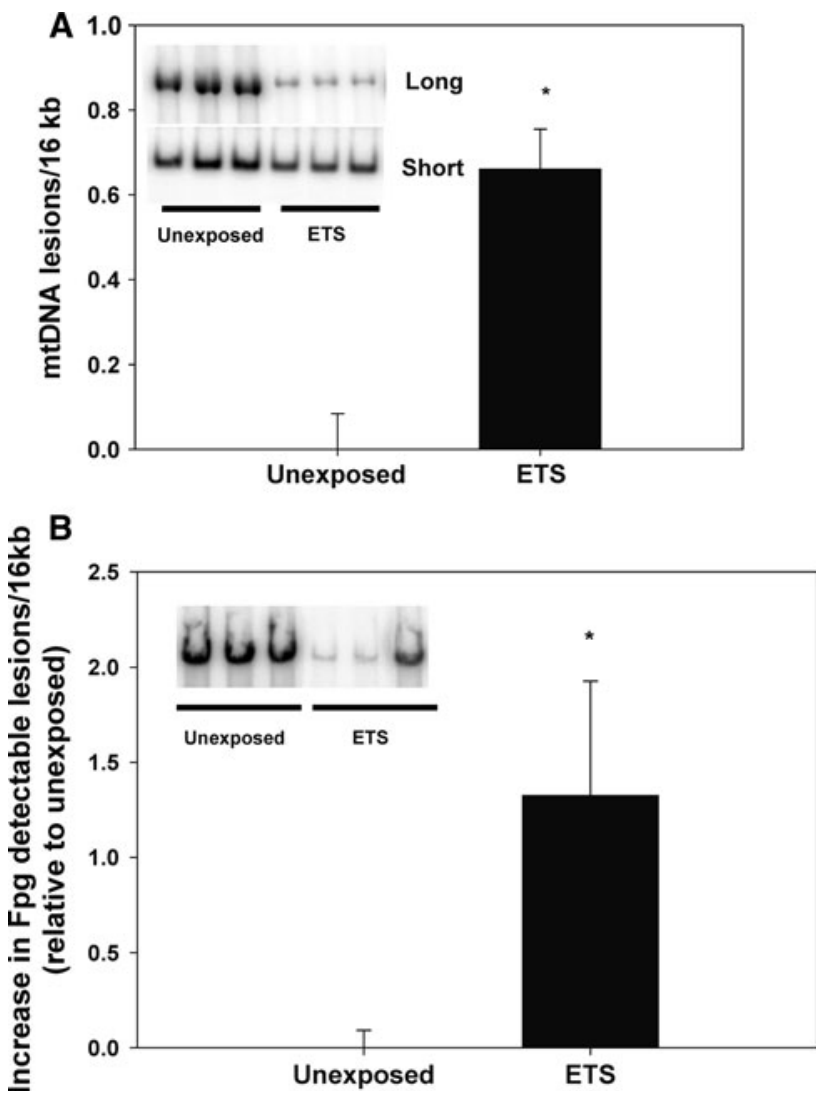

Fig. 3 Mitochondrial DNA ( $m t D N A)$ damage in M. mulatta aorta. MtDNA damage was quantified from genomic DNA preparations extracted from aorta. a mtDNA damage determined by QPCR; ( $n=3$ /group/tissue). The inset (lanes $1-3$ are unexposed control, lanes 4-6 are ETS exposed) shows the full-length QPCR product (Long) that is used to quantify relative levels of mtDNA damage (less product indicates increased damage) relative to control, whereas the lower inset (lanes 1-3 are unexposed control, lanes 4-6 are ETS exposed) shows the smaller QPCR product (Short) that is used for mtDNA copy number normalization. Bar graph shows the relative level of mtDNA damage (normalized for copy number) between exposure groups. b mtDNA damage detected by Fpg digestion followed by QPCR. The inset shows QPCR results for Fpg-treated unexposed controls and ETS-exposed animals (lanes 1-3 and 4-6, respectively), showing the full-length QPCR product (less product indicates increased mtDNA damage). The bar graph shows the difference in Fpg detectable lesions $( \pm$ Fpg) in ETS-exposed monkeys relative to unexposed controls. Unexposed, $M$. mulatta age-matched control; ETS, M. mulatta exposed to $1 \mathrm{mg} / \mathrm{m}^{3}$ ETS during gestation to 1 year of age. Asterisks indicate significant difference $(P \leq 0.05)$ from unexposed $M$. mulatta

\section{Discussion}

Perinatal exposure to ETS has been associated with altered physiologic and developmental changes, which can also be related to increased risk for childhood and adult disease development $[1,2,18,19]$. Previous studies have shown that gestational exposure to cigarette smoke increases individual risk for atherogenesis in rodents [1], which was accompanied by increased mitochondrial damage and 

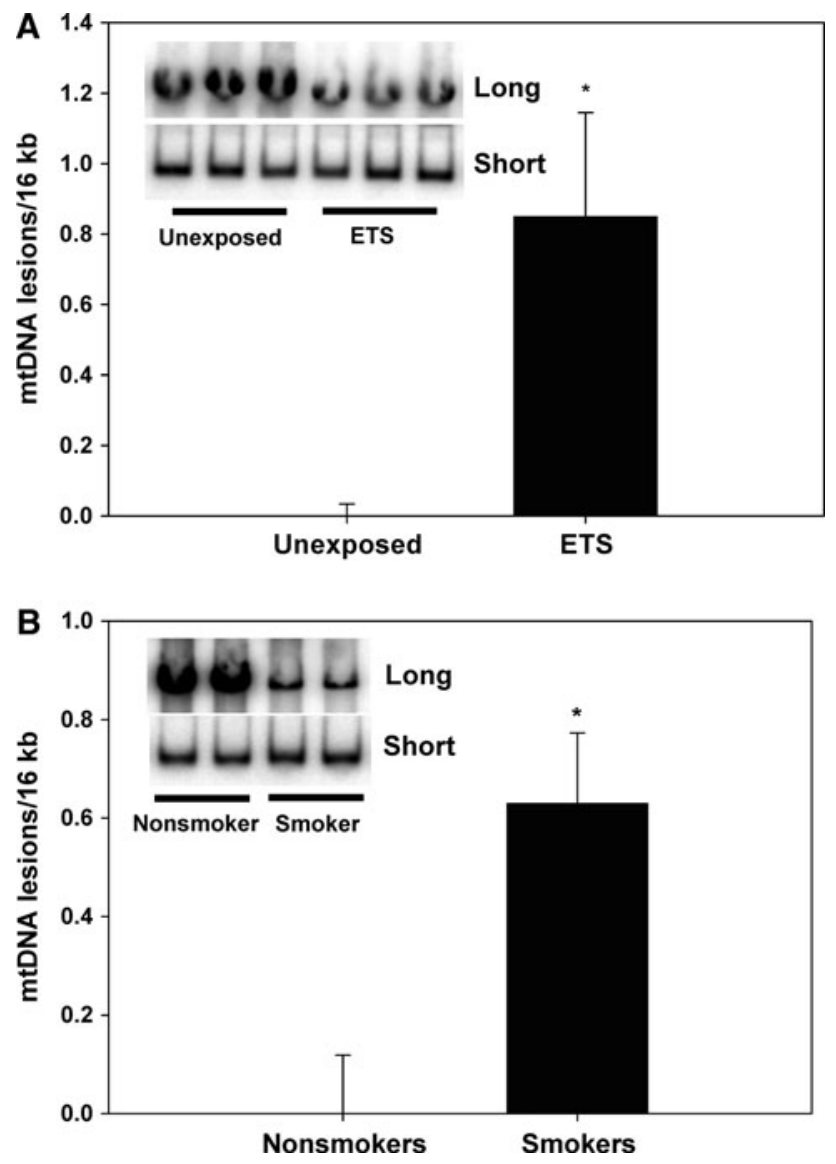

Fig. 4 Mitochondrial DNA ( $m t D N A)$ damage in M. mulatta and human buffy coat tissues. MtDNA damage was quantified using QPCR on genomic DNA preparations extracted from M. mulatta and human buffy coats. a Bar graph showing the relative level of mtDNA damage (normalized for copy number) in $M$. mulatta perinatally exposed the ETS compared to unexposed controls. The inset (lanes 1-3 are unexposed control, lanes 4-6 are ETS exposed) shows the full-length QPCR product (Long) which is used to quantify relative levels of mtDNA damage (less product indicates increased damage) relative to control, whereas the lower inset (lanes 1-3 are unexposed control, lanes 4-6 are ETS exposed) shows the smaller QPCR product (Short) that is used for mtDNA copy number normalization, b Bar graph showing the relative level of mtDNA damage (normalized for copy number) in human smokers $(N=8)$ compared to non-smokers $(N=5)$. The inset (lanes 1, 2 are non-smoker control, lanes 3, 4 are smoker) shows the full-length QPCR product (Long) that is used to quantify relative levels of mtDNA damage (less product indicates increased damage) relative to control, whereas the lower inset (lanes 1, 2 are non-smoker control, lanes 3, 4 are smoker) shows the smaller QPCR product (Short) that is used for mtDNA copy number normalization. Asterisks indicate significant difference $(P<0.05)$

sensitivity to inhibitors of mitochondrial function [2]. The studies herein represent an extension of those reports using archived vascular tissues (aorta) from the non-human primate, M. mulatta. Here, it is shown that M. mulatta exposed to low levels of ETS during gestation to early childhood resulted in significantly increased oxidative stress, mitochondrial dysfunction and damage in vascular

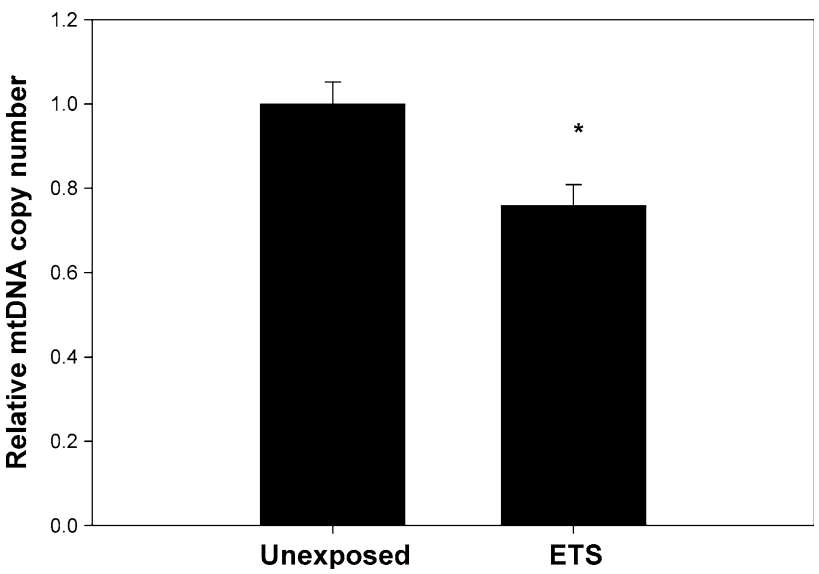

Fig. 5 Mitochondrial DNA ( $m t D N A)$ copy number in M. mulatta. MtDNA copy numbers were quantified using QPCR of the short QPCR fragment from $15 \mathrm{ng}$ genomic DNA extracted from aorta as template, as shown in 3A, lower inset (Short). Unexposed, M. mulatta age-matched control; ETS, $M$. mulatta exposed to $1 \mathrm{mg} / \mathrm{m}^{3}$ ETS during gestation to 1 year of age. Asterisks indicate significant difference $(P<0.05)$ from unexposed $M$. mulatta $(n=3 /$ group/ tissue)

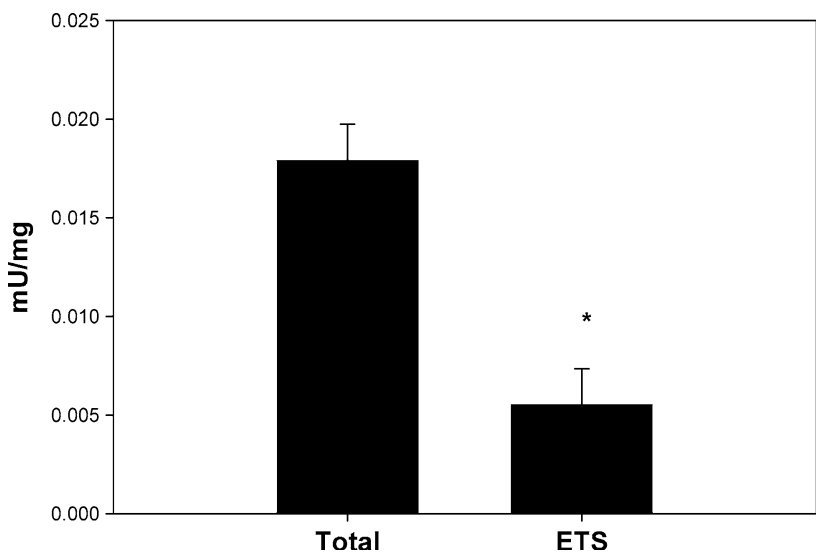

Fig. 6 Cytochrome oxidase (complex IV) activity in M. mulatta. Complex IV activity was determined from mitochondrial isolates extracted from aortic tissues. Unexposed, $M$. mulatta age-matched control; ETS, $M$. mulatta exposed to $1 \mathrm{mg} / \mathrm{m}^{3}$ ETS during gestation to 1 year of age. Asterisks indicate significant difference $(P<0.05)$ from unexposed $M$. mulatta ( $n=3 /$ group/tissue)

tissue (aorta). These effects were also accompanied by significantly decreased mitochondrial antioxidant capacity and mtDNA copy numbers in vascular tissue. Increased mitochondrial damage was also detected in buffy coat tissues in exposed M. mulatta, suggesting its potential as a biomarker for exposure in a surrogate tissue. Similarly, quantification of mtDNA damage in buffy coat tissues from human smokers and non-smokers showed that smokers had significantly increased mtDNA damage compared to nonsmokers, consistent with the concept that tobacco smoke exposure increases mitochondrial damage. Overall, these studies provide molecular evidence for increased oxidative 

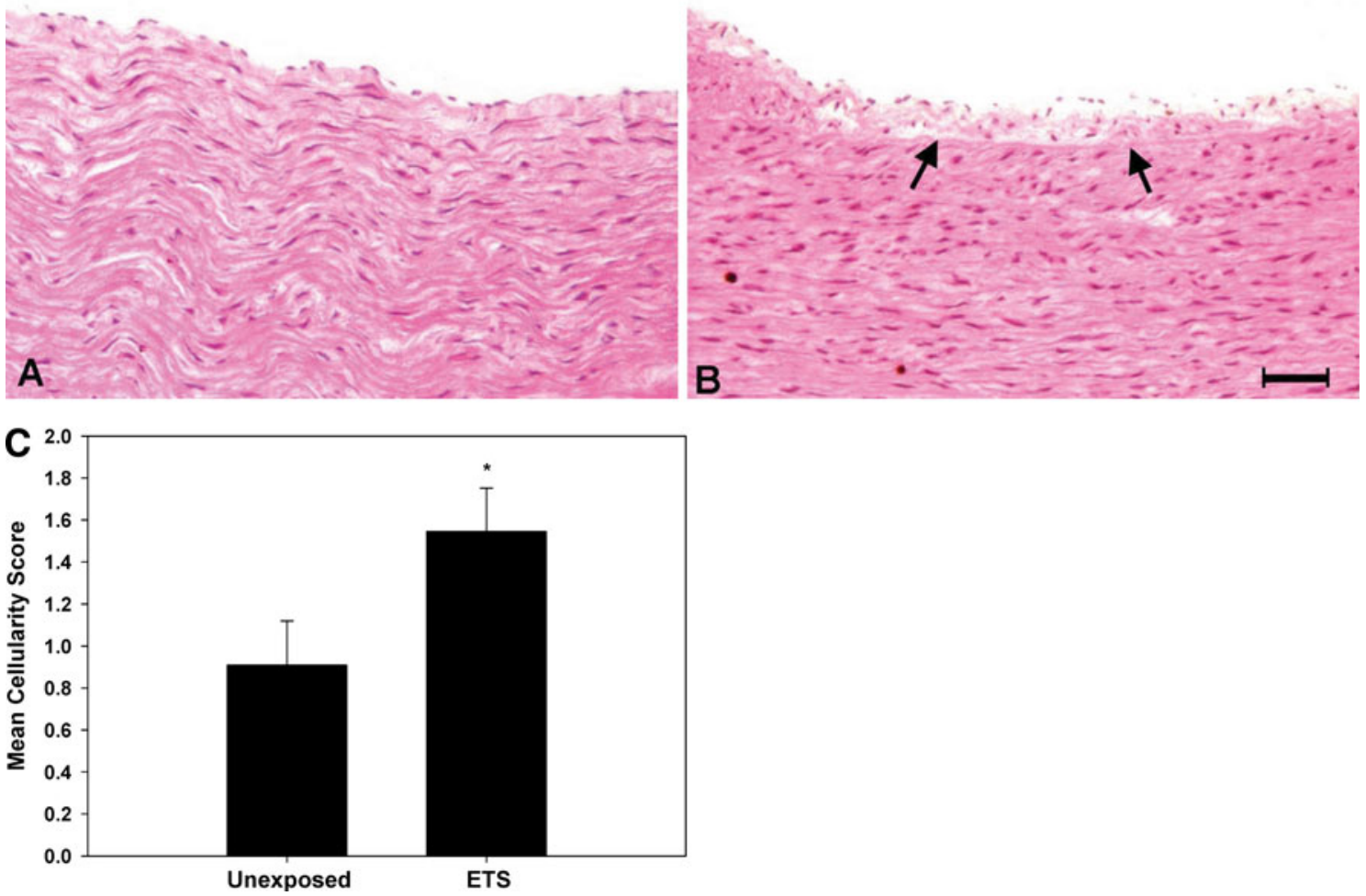

Fig. 7 Histopathology of abdominal aorta from M. mulatta. Histopathology of H\&E-stained section of abdominal aorta shows accumulation of inflammatory cells with widening of the subintimal region (arrows) in the ETS-exposed animal (b) versus age-matched

stress and mitochondrial damage in primate aortic tissues associated with perinatal ETS exposure and supports the hypothesis that the cardiovasculature is prone to mitochondrial damage and dysfunction in response to tobacco smoke exposure in primates.

A limitation of the current study was that the duration of ETS exposure encompassed both in utero and early childhood development, and thus, was perinatal. Hence, with these tissues, it was not possible to distinguish the effects of gestational versus neonatal, early childhood ETS exposure. Previous studies have shown that in utero tobacco smoke exposure significantly influences mitochondrial damage and disease development; consequently, it is possible that the effects observed may be primarily due to gestational events, although other reports clearly show that adult exposure to tobacco smoke also cause mitochondrial damage, dysfunction and increased disease development $[1,2,12,20-22]$. Further studies are required to delineate the cardiovascular effects of fetal versus neonatal exposure to tobacco smoke. While this report did not specifically evaluate atherosclerotic lesion development because the monkeys were not fed atherogenic diets and were still relatively young ( 1 year) at the end of the study, basic histological analyses did suggest early atherogenic changes in aortas of $M$. mulatta exposed to perinatal ETS. Previous control aorta (a). Magnification bar (inset on panel b) is $50 \mu \mathrm{M}$. Bar graph (c) depicts mean cellularity score \pm SEM (see "Methods"), showing that a significant difference exists between unexposed and ETS-exposed (perinatal) animals

studies in hypercholesterolemic rodents have shown an association between perinatal CVD risk factor exposure and increased CVD [1, 2, 23], and other studies have shown that mtDNA damage is significantly increased human atherosclerotic tissues, and moreover, that mtDNA damage occurs prior to, or, coincident with atherogenesis $[24,25]$. Hence, the observations of increased oxidative and nitrative stress, plus mitochondrial damage and dysfunction occurring in vascular tissues harvested from nonhuman primates exposed to perinatal ETS are features also found in human atherosclerotic tissues. In these studies, mtDNA damage was also significantly increased in blood, suggesting that perinatal ETS exposure impacted the mitochondrial genome in multiple tissues. Although mtDNA integrity has not been specifically examined in blood from children exposed to ETS, previous studies on using umbilical cord bloods from infants of smoking mothers have shown increased frequency of genomic deletions in nuclear DNA, mediated by $\mathrm{V}(\mathrm{D}) \mathrm{J}$ recombinase [26]. Similarly, analysis of bronchoalveolar lavage tissues collected from smokers revealed a significant increase in both nuclear and mitochondrial genetic damage compared to non-smokers [20], consistent with the findings in buffy coats samples from human smokers in this study. While the impacts of this genetic damage are not clearly known, they 
likely affect aspects of mitochondrial function, and thus, cellular function.

MtDNA copy number was decreased $\sim 25 \%$ in aortic tissue from $M$. mulatta exposed to perinatal ETS. Decreased mtDNA copy numbers associated with cigarette smoke exposure have been previously noted in bronchoalveolar lavage tissues from smokers [20]. Similarly, depletion of mtDNA copy numbers have been noted in tissues (heart and skeletal muscle) from adults on zidovudine (AZT) therapy, and from infant primates exposed to AZT in utero [27-29]. A previous study investigating genomic DNA levels in brain tissues from the same monkeys used in these studies reported regional variation of DNA content compared to controls[30]; while those studies did not specifically examine mtDNA levels, they are consistent with tissue variances in DNA content between M. mulatta exposed to perinatal ETS compared to controls. It is possible that components of ETS capable of inducing damage and/or covalent association with genomic DNA, or more specifically, the mtDNA would contribute to mtDNA depletion by blocking the mitochondrial-specific polymerase $\gamma$ and/or causing damage to the template strand, thus interfering with its replication. Interestingly, no mtDNA copy number differences were observed in blood buffy coat genomic DNAs in M. mulatta or human smokers; these differences between tissues may relate to their characteristics and tolerance for mitochondrial dysfunction. Vascular tissue is relatively quiescent compared to buffy coat (leukocytes and platelets), it may be that mtDNA copy numbers can be more readily maintained in dividing cells compared to quiescent tissues. Alternatively or additionally, vascular tissue may be more sensitive to decreased mitochondrial function relative to blood tissues, and hence, less tolerant of mitochondrial damage and therefore more prone to cell loss/death with increased mtDNA damage. Because mtDNA damage was higher in blood tissue than aorta compared to matched controls $(0.85 \pm 0.29$ lesions/ $16 \mathrm{~kb}$ vs. $0.66 \pm 0.09$ lesions $/ 16 \mathrm{~kb}$, respectively), this suggests that blood cells may tolerate higher levels of mtDNA damage compared to vascular cells, and therefore may not be lost to the same degree. This, plus an increased replicative potential, may allow maintenance of similar mtDNA copy numbers between exposed and controls in buffy coat tissues.

Previous studies utilizing tissues from the same M. mulatta used in these studies have reported altered cytokine response and immune effectors in the circulation and the lung [31, 32], hence it is clear that perinatal exposure to a low dose of ETS has multi-organ impacts, including the vasculature. Additionally, this and other studies have shown that the mitochondrion is a vulnerable target of ETS exposure in both the adult and developmental exposure models $[1,2,12,20,22,33-36]$. Numerous reports have shown that mitochondria are sensitive to both reactive oxygen- and nitrogen-mediated damage and alterations in function[11, 37-43]. This susceptibility of the mtDNA to damage is thought to be due to several factors, including the lack of both protective histone proteins and the attachment of the mtDNA to the matrix side of the mitochondrial inner membrane, placing it in proximity to reactive lipophilic species and reactive lipid oxidation products (generated within the membrane) that are capable of modifying the mtDNA [44]. Early injury to mitochondria mediated by prenatal and/or childhood exposure may hasten the onset or increase the risk for a variety of diseases, including CVD. To our knowledge, this is the first report showing that perinatal ETS exposure in a nonhuman primate model results in significant increases in mitochondrial damage and function. The findings of this study are consistent with previous reports in rodents that reveal increased mitochondrial damage with developmental environmental toxicant exposure, and thus, are consistent with the hypothesis that the mitochondrion is a vulnerable cellular target to environmental toxicants. Because the mitochondrion is an organelle essential for multiple cellular functions, sustained mitochondrial damage likely increases the likelihood of cellular dysfunction, thereby increasing risk for disease development.

Acknowledgments This work was supported by NIH grants HL77419 and HL94518 (SWB) and by Philip Morris USA Inc (SWB).

Open Access This article is distributed under the terms of the Creative Commons Attribution Noncommercial License which permits any noncommercial use, distribution, and reproduction in any medium, provided the original author(s) and source are credited.

\section{References}

1. Yang, Z., Knight, C. A., Mamerow, M., Vickers, K., Penn, A., Postlethwait, E., \& Ballinger, S. W. (2004). Prenatal environmental tobacco smoke exposure promotes adult atherogenesis and mitochondrial damage in apoE-/- mice fed a chow diet. Circulation, 110, 3715-3720.

2. Yang, Z., Harrison, C. M., Chuang, G., \& Ballinger, S. W. (2007). The role of tobacco smoke induced mitochondrial damage in vascular dysfunction and atherosclerosis. Mutation Research, 621, 61-74.

3. American Heart Association. (2006). Heart disease and stroke statistics-2006 update. Circulation, 105, 1-67.

4. Guyer, B., Hoyert, D. L., Martin, J. A., Ventura, S. J., MacDorman, M. F., \& Strobino, D. M. (1999). Annual summary of vital statistics-1998. Pediatrics, 104, 1229-1246.

5. Butler, N., Goldstein, H., \& Ross, E. (1972). Cigarette smoking in pregnancy: its influence on birth weight and perinatal mortality. British Medical Journal, 2, 127-130.

6. England, L. J., Kendrick, J. S., Gargiullo, P. M., Zahniser, S. C., \& Hannon, W. H. (2001). Measures of maternal tobacco exposure and infant birth weight at term. American Journal of Epidemiology, 153, 954-960. 
7. Tarantal, A., \& Hendrickx, A. (1988). Use of ultrasound for early pregnancy detection in the rhesus and cynomolgus macaque (Macaca mulatta and Macaca fascicularis). Journal of Medical Primatology, 17, 105-112.

8. Tarantal, A., \& Hendrickx, A. (1988). Prenatal growth in the cynomolgus and rhesus macaque: A comparison by ultrasonography. American Journal of Primatology, 15, 309-323.

9. Teague, S., Pinkerton, K., Goldsmith, M., Gebremichael, A., Chang, S., Jenkins, R., et al. (1994). Sidestream cigarette smoke generation and exposure system for environmental tobacco smoke studies. Inhalation Toxicology, 6, 79-93.

10. Flohe, L., \& Otting, F. (1984). Superoxide dismutase assays. Methods in Enzymology, 105, 93-104.

11. Ballinger, S. W., Patterson, W. C., Yan, C.-N., Doan, R., Burow, D. L., Young, C. G., et al. (2000). Hydrogen peroxide and peroxynitrite induced mitochondrial DNA damage and dysfunction in vascular endothelial and smooth muscle cells. Circulation Research, 86, 960-966.

12. Knight-Lozano, C. A., Young, C. G., Burow, D. L., Hu, Z., Uyeminami, D., Pinkerton, K., et al. (2002). Cigarette smoke exposure and hypercholesterolemia increase mitochondrial damage in cardiovascular tissues. Circulation, 105, 849-854.

13. Wallace, D. C., Garrison, K., \& Knowler, W. (1985). Dramatic founder effects in Amerindian mitochondrial DNAs. American Journal of Physical Anthropology, 68, 149-155.

14. Ballinger, S. W., Van Houten, B., Jin, G. F., Conklin, C. A., \& Godley, B. F. (1999). Hydrogen peroxide causes significant mitochondrial DNA damage in human RPE cells. Experimental Eye Research, 68, 765-772.

15. Levine, R. L., Williams, J. A., Stadtman, E. R., \& Shacter, E. (1994). Carbonyl assays for determination of oxidatively modified proteins. Oxygen Radicals in Biological Systems, 233(Pt C), 346-357.

16. Trounce, I., Kim, Y. L., Jun, A. S., \& Wallace, D. C. (1996). Assessment of mitochondrial oxidative phosphorylation in patient muscle biopsies, lymphoblasts, and transmitochondrial cell lines. Methods in Enzymology, 264, 484-509.

17. Lowry, O. H., Rosebrough, N. J., Farr, A. L., \& Randall, R. J. (1951). Protein measurement with the folin phenol reagent. The Journal of Biological Chemistry, 193, 265-275.

18. Holberg, C., Wright, A., Martinez, F., Morgan, W., \& Taussig, L. (1993). Child day care, smoking by caregivers and lower respiratory tract illness in the first 3 years of life. Pediatrics, 91, 885-892.

19. Witschi, H., Joad, J. P., \& Pinkerton, K. (1997). The toxicology of environmental tobacco smoke. Annual Review of Pharmacology and Toxicology, 37, 29-52.

20. Ballinger, S. W., Bouder, T. G., Davis, G. S., Judice, S. A., Nicklas, J. A., \& Albertini, R. J. (1996). Mitochondrial genome damage associated with cigarette smoking. Cancer Research, 56, 5692-5697.

21. Cakir, Y., Yang, Z., Knight, C. A., Pompilius, M., Westbrook, D., Bailey, S. M., et al. (2007). Effect of alcohol and tobacco smoke on mtDNA damage and atherogenesis. Free Radical Biology and Medicine, 43, 1279-1288.

22. Bailey, S. M., Mantena, S. K., Millender-Swain, T., Cakir, Y., Jhala, N. C., Chhieng, D., et al. (2009). Ethanol and tobacco smoke increase hepatic steatosis and hypoxia in the hypercholesterolemic apoE(-/-) mouse: Implications for a "multihit" hypothesis of fatty liver disease. Free Radical Biology and Medicine, 46, 928-938.

23. Napoli, C., De Nigris, F., Welch, J. S., Calara, F. B., Stuart, R. O., Glass, C. K., et al. (2002). Maternal hypercholesterolemia during pregnancy promotes early atherogenesis in LDL receptor-deficient mice and alters aortic gene expression determined by microarray. Circulation, 105, 1360-1367.
24. Ballinger, S. W., Patterson, C., Knight-Lozano, C. A., Burow, D. L., Conklin, C. A., Hu, Z., et al. (2002). Mitochondrial integrity and function in atherogenesis. Circulation, 106, 544-549.

25. Beckman, J. S., Ye, Y. Z., Anderson, P. G., Chen, J., Accavitti, M. A., Tarpey, M. M., et al. (1994). Extensive nitration of protein tyrosines in human atherosclerosis detected by immunohistochemistry. Biological Chemistry Hoppe-Seyler, 375, 81-88.

26. Finette, B. A., O'Neill, J. P., Vacek, P. M., \& Albertini, R. J. (1998). Gene mutations with characteristic deletions in cord blood $\mathrm{T}$ lymphocytes associated with passive maternal exposure to tobacco smoke. Nature Medicine, 4, 1144-1151.

27. Arnaudo, E., Dalakas, M., Shanske, S., Moraes, C. T., DiMauro, S., \& Schon, E. A. (1991). Depletion of muscle mitochondrial DNA in AIDS patients with zidovudine-induced myopathy. Lancet, 337, 508-510.

28. Gerschenson, M., Erhart, S. W., Paik, C. Y., Claire, M. C. S., Nagashima, K., Skopets, B., et al. (2000). Fetal mitochondrial heart and skeletal muscle damage in Erythrocebus patas monkeys exposed in utero to 3 '-azido-3 '-deoxythymidine. AIDS Research and Human Retroviruses, 16, 635-644.

29. Gerschenson, M., \& Poirier, M. C. (2000). Fetal patas monkeys sustain mitochondrial toxicity as a result of in utero zidovudine exposure. Prevention and Treatment of Hiv Infection in Infants and Children, 918, 269-281.

30. Slotkin, T. A., Pinkerton, K. E., \& Seidler, F. J. (2006). Perinatal environmental tobacco smoke exposure in rhesus monkeys: Critical periods and regional selectivity for effects on brain cell development and lipid peroxidation. Environmental Health Perspectives, 114, 34-39.

31. Wang, L., Joad, J. P., Abel, K., Spinner, A., Smiley-Jewell, S., Liu, H., et al. (2007). Effects of environmental tobacco smoke on the developing immune system of infant monkeys. Journal of Allergy and Clinical Immunology, 120, 445-451.

32. Wang, L., Joad, J. P., Zhong, C. Y., \& Pinkerton, K. E. (2008). Effects of environmental tobacco smoke exposure on pulmonary immune response in infant monkeys. Journal of Allergy and Clinical Immunology, 122, 400-406.

33. Chuang, G. C., Yang, Z., Westbrook, D. G., Pompilius, M., Ballinger, C. A., White, C. R., et al. (2009). Pulmonary ozone exposure induces vascular dysfunction, mitochondrial damage, and atherogenesis. American Journal of Physiology-Lung Cellular and Molecular Physiology, 297, L209-L216.

34. Gvozdjakova, A., Bada, V., Sany, L., Kucharska, J., Kruty, F., Bozek, P., et al. (1984). Smoke cardiomyopathy: disturbance of oxidative processes in myocardial mitochondria. Cardiovascular Research, 18, 229-232.

35. Gvozdjakova, A., Kucharska, J., \& Gvozdjak, J. (1992). Effect of smoking on the oxidative processes of cardiomyocytes. Cardiology, 1992, 81-84.

36. Gvozdjakova, A., Simko, F., Kucharska, J., Braunova, Z., Psenek, P., \& Kyselovic, J. (1999). Captopril increased mitochondrial coenzyme $\mathrm{Q}_{10}$ level, improved respiratory chain function and energy production in the left ventricle in rabbits with smoke mitochondrial cardiomyopathy. BioFactors, 10, 61-65.

37. Turrens, J. F. (2003). Mitochondrial formation of reactive oxygen species. Journal of Physiology, 552, 335-344.

38. Cassina, A., \& Radi, R. (1996). Differential inhibitory action of nitric oxide and peroxynitrite on mitochondrial electron transport. Archives of Biochemistry and Biophysics, 328, 309-316.

39. Cassina, A. M., Hodara, R., Souza, J. M., Thomson, L., Castro, L., Ischiropoulos, H., et al. (2000). Cytochrome c nitration by peroxynitrite. The Journal of Biological Chemistry, 275, 21409-21415.

40. Yan, L.-J., \& Sohal, R. S. (1998). Mitochondrial adenine nucleotide translocase is modified oxidatively during aging. 
Proceedings of the National Academy of Sciences of the United States of America, 95, 12896-12901.

41. Yakes, F. M., \& Van Houten, B. (1997). Mitochondrial DNA damage is more extensive and persists longer than nuclear DNA damage in human cells following oxidative stress. Proceedings of the National Academy of Sciences of the United States of America, 94, 514-519.

42. MacMillan-Crow, L. A., Crow, J. P., \& Thompson, J. A. (1998). Peroxynitrite-mediated inactivation of manganese superoxide dismutase involves nitration and oxidation of critical gyrosine residues. Biochemistry, 37, 1613-1622.
43. Duan, J., \& Karmazyn, M. (1989). Relationship between oxidative phosphorylation and adenine nucleotide translocase activity in two populations of cardiac mitochondria and mechanical recovery of ischemic hearts following reperfusion. Canadian Journal of Physiology and Pharmacology, 67, 704-709.

44. Bandy, B., \& Davison, A. J. (1990). Mitochondrial mutations may increase oxidative stress: implications for carcinogenesis and aging? Free Radicals of Biology Medicine, 8, 523-539. 